\title{
Cross-polarization suppression in C-shaped microstrip patch antenna employing anisotropic dielectrics
}

\author{
Hongyu Shi**, Shitao Zhu*, Jianxing $\mathrm{Li}^{*}$, Anxue Zhang* ${ }^{*} \S$ and Zhuo $\mathrm{Xu}^{\dagger}$ \\ *School of Electronic and Information Engineering, Xi'an Jiaotong University \\ Xi'an, Shaanxi 710049, P. R. China \\ ${ }^{\dagger}$ Electronic Materials Research Laboratory and the Key Laboratory of the Ministry of Education \\ Xi'an Jiaotong University, Xi'an, Shaanxi 710049, P. R. China \\ †honyo.shi1987@gmail.com \\ §anxuezhang@mail.xjtu.edu.cn
}

Received 13 June 2017; Revised 20 July 2017; Accepted 28 July 2017; Published 23 August 2017

\begin{abstract}
An anisotropic dielectric realized by layered ceramic structures was adopted to design a low cross-polarization C-shaped patch antenna. The anisotropic dielectric performs as a substrate and can cause additional cross-polarized fields which are able to cancel the cross-polarized fields generated by the $\mathrm{C}$-shaped patch itself, and then reduce the cross-polarization level. Compared to the $\mathrm{C}$-shaped patch antenna with an isotropic substrate, the cross-polarization of the proposed antenna is suppressed by more than $15 \mathrm{~dB}$ with a little gain enhancement at $2.4 \mathrm{GHz}$. The anisotropic dielectric has a little impact on the direction of the C-shaped patch antenna. The gain of the proposed C-shaped patch antenna is $6.8 \mathrm{~dB}$ with a cross-polarization of $-28 \mathrm{~dB}$.
\end{abstract}

Keywords: Anisotropic dielectrics; layered ceramic structures; low cross-polarization; patch antennas.

\section{Introduction}

Patch antennas are widely used in various wireless communication systems, ${ }^{1}$ such as base stations, Wi-Fi and body area networks. Nowadays, people are seeking patch antennas with smaller size with lower cross-polarization. For symmetric rectangular or circular patch antennas, the crosspolarized radiation results from the higher mode of the patch (e.g., the $\mathrm{TM}_{02}$ mode). Different techniques have been developed to suppress the cross-polarization of symmetric patch antennas.

Defected ground structures (DGS) are often used to reduce the cross-polarization component of the rectangular or circular patch antennas. Dot- and arc-shaped DGS have been used in circular patch for a relative cross-polarization suppression around $10 \mathrm{~dB} .{ }^{2,3}$ In Ref. 4, an L-shaped DGS is used to reduce the cross-polarization by $15 \mathrm{~dB}$. Although these examples demonstrate that DGS can effectively suppress the cross-polarization of the symmetric patch antenna, the DGS method only works in the $H$-plane, and will reduce the gain and front-back ratio.

With an additional metal wall ${ }^{5}$ and spiral-ring resonator structure, ${ }^{6}$ the cross-polarization of the E-shaped patch antennas can be reduced. By using the overlapped scheme, Zhou et al. designed a low cross-polarization patch antenna array. ${ }^{7}$ In addition, the cross-polarization level can also be degraded by introducing shorting pins in a rectangular patch antenna. ${ }^{8}$ However, such an approach still can only reduce the cross component in the $H$-plane.

Although the cross-polarization component can be reduced in patch antennas by the above methods, these methods are only useful for suppressing the cross-polarization component raised by the higher resonate mode in symmetric patch antennas. With an asymmetric C-shaped topology, the size of the patch antennas can be significantly reduced ${ }^{9}$ which is highly desirable in compact wireless communication and body area networks. However, due to the asymmetric topology, C-shaped patch antennas suffer a relatively high crosspolarization which severely limits its applications. ${ }^{10}$ More over, such a high cross-polarization level can hardly be mitigated by DGS or other techniques using symmetric structures or isotropic materials.

In this paper, by implementing an anisotropic substrate, additional cross-polarized electric field is induced, with opposite phase to the cross-polarization component generated by the asymmetric C-shaped topology. Thus, the overall cross-polarization level of the C-shaped patch antenna can be significantly suppressed. Such anisotropic dielectrics can be realized by stacking two materials of different permmitivities periodically. ${ }^{11}$ To the best of our knowledge, this is the first time for anisotropic dielectrics to be applied in the demonstration of cross-polarization level suppression.

$\$$, Corresponding authors.

This is an Open Access article published by World Scientific Publishing Company. It is distributed under the terms of the Creative Commons Attribution 4.0 (CC-BY) License. Further distribution of this work is permitted, provided the original work is properly cited. 


\section{Design of the Low Cross-Polarized C-Shaped Patch Antenna}

Anisotropic mediums and metamaterials are widely used to control the polarization of electromagnetic waves. ${ }^{12-15}$ Due to the anisotropy of the substrate, the polarization of the fields in rectangular patch antennas can be tuned as shown in Fig. 1. With an isotropic substrate, the $y$-polarized fields at the two edges are symmetric and cancel each other as shown in Fig. 1(a). However, compared to Fig. 1(a), in Fig. 1(b), on the left edge, the $y$ component of the electric fields is smaller, meanwhile, on the right edge, the $-y$ component of the electric fields is bigger. Thus, with an anisotropic substrate, the $y$-polarized fields at the edges are asymmetric and the additional net $-y$ component field is raised. Such additional fields can used to cancel the cross-polarized fields generated by the asymmetric topology of the $\mathrm{C}$-shaped patch antenna.

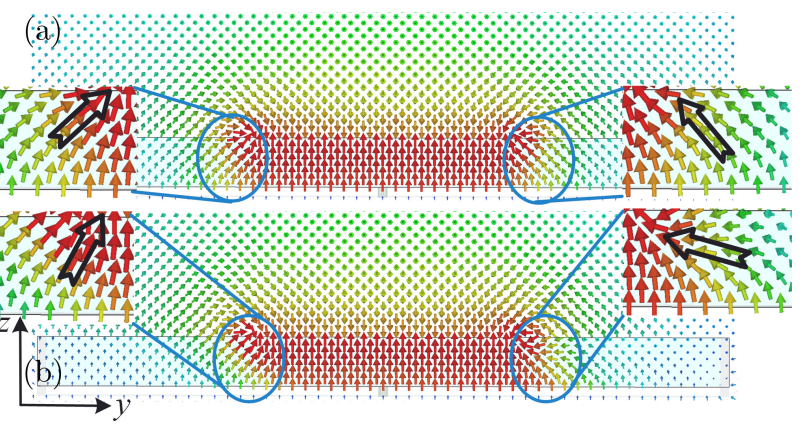

Fig. 1. Electronic fields in rectangular patch antenna: (a) with isotropic substrate; (b) with anisotropic substrate.

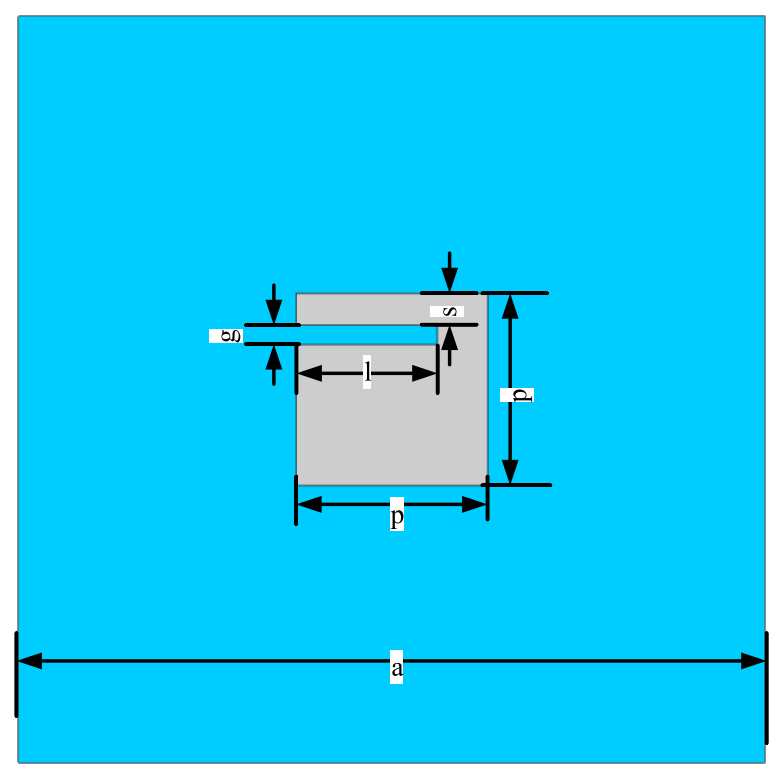

(a)
Then, the cross-polarization level can be suppressed effectively.

The dimensions of the designed C-shaped patch antenna is shown in Fig. 2. The blue area is the anisotropic substrate and the gray area is the ground plane. The geometric parameters are set as: $a=70 \mathrm{~mm}, p=18 \mathrm{~mm}, l=13.3 \mathrm{~mm}, s=3 \mathrm{~mm}$, $g=1.8 \mathrm{~mm}$ and $f=32.6 \mathrm{~mm}$. The permittivity of the anisotropic substrate $\overline{\bar{\epsilon}}$ is

$$
\overline{\bar{\epsilon}}=\left(\begin{array}{ccc}
7.0 & 0 & 0 \\
0 & 4.1 & -2.9 \\
0 & -2.9 & 4.1
\end{array}\right) .
$$

Such an anisotropic dielectric can be realized by a layered structure as shown in Fig. 3. This structure contains foam layers with $\epsilon_{1}=1$ and ceramic layers with $\epsilon_{2}=36$. The thicknesses of the foam and ceramic layers are $d_{1}=2.9 \mathrm{~mm}$ and $d_{2}=0.6 \mathrm{~mm}$, respectively. The effective permittivity tensor $\overline{\bar{\epsilon}}^{\prime}$ in the $45^{\circ}$ rotated axis $\left(x^{\prime}, y^{\prime}, z^{\prime}\right)$ of such a structure can be calculated by ${ }^{11}$

$$
\begin{gathered}
\epsilon_{x^{\prime}}=\epsilon_{y^{\prime}}=\frac{\epsilon_{1}+\eta \epsilon_{2}}{1+\eta}=7, \\
\frac{1}{\epsilon_{z^{\prime}}}=\frac{1}{1+\eta}\left(\frac{1}{\epsilon_{1}}+\frac{\eta}{\epsilon_{2}}\right)=\frac{1}{1.2},
\end{gathered}
$$

where

$$
\eta=\frac{d_{1}}{d_{2}}
$$

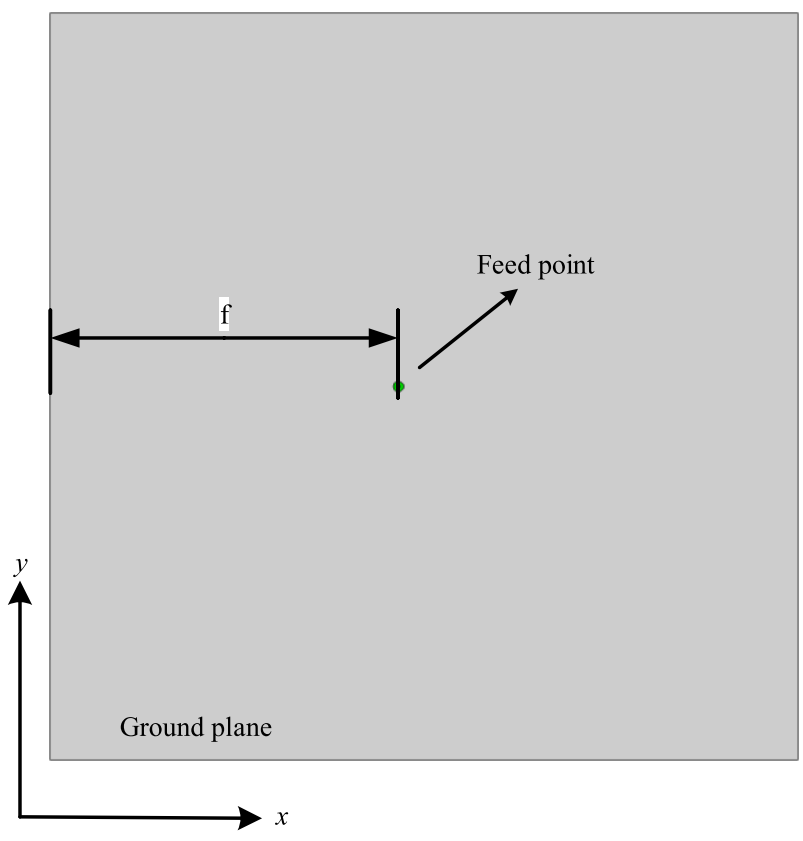

(b)

Fig. 2. The topology of the designed C-shaped patch antenna: (a) top view; (b) back view. 


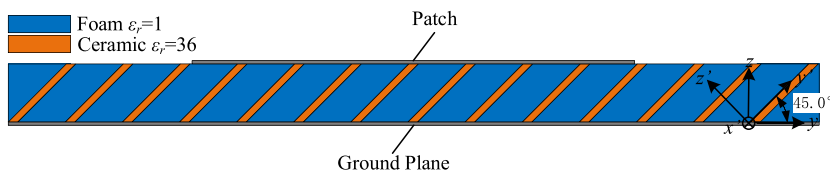

Fig. 3. The C-shaped patch antenna with layered anisotropic structure.

Thus, $\overline{\bar{\epsilon}}$ can be calculated from

$$
\overline{\bar{\epsilon}}=\frac{R \overline{\overline{\epsilon^{\prime}}} R^{T}}{\operatorname{det}(\mathrm{R})},
$$

where $R$ is the axial transform matrix

$$
R=\left(\begin{array}{ccc}
1 & 0 & 0 \\
0 & \frac{\sqrt{2}}{2} & \frac{\sqrt{2}}{2} \\
0 & -\frac{\sqrt{2}}{2} & \frac{\sqrt{2}}{2}
\end{array}\right) .
$$

To demonstrate the cross-polarization suppression performance of the proposed method, a C-shaped patch antenna with the same dimensions as shown in Fig. 2 and an isotropic substrate is designed for comparison. The permittivity of its isotropic substrate is 4.4 .

\section{Simulation Results}

The numerical models of C-shaped patch antennas with isotropic and anisotropic substrates were set up and simulated in a commercial CST MICROWAVE STUDIO. The impedance of the waveguide port is set as $50 \Omega$.

Figure 4 shows the simulated $\left|S_{11}\right|$ results of the C-shaped patch antennas with isotropic and anisotropic substrates. The resonate frequency of these $\mathrm{C}$-shaped patch antennas is 2.4 GHz. The simulated $\left|S_{11}\right|$ are $-19 \mathrm{~dB}$ and $-18.7 \mathrm{~dB}$ for

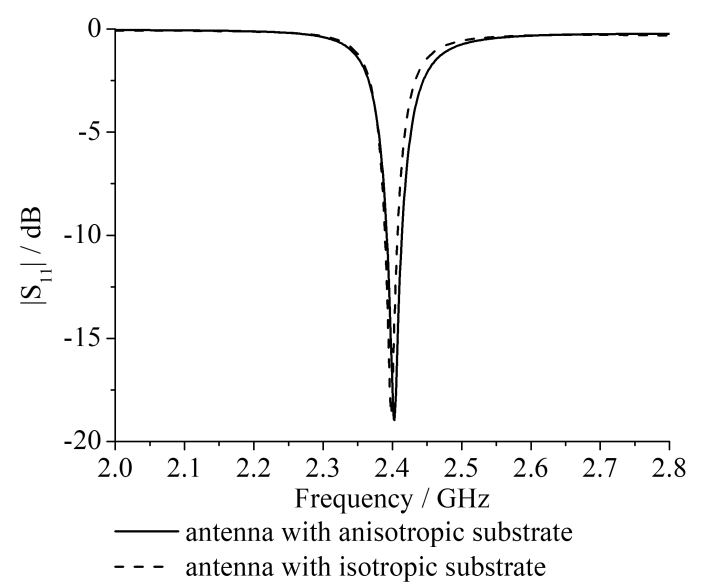

Fig. 4. The simulated $\left|S_{11}\right|$ of the designed C-shaped patch antennas with isotropic and anisotropic substrates.

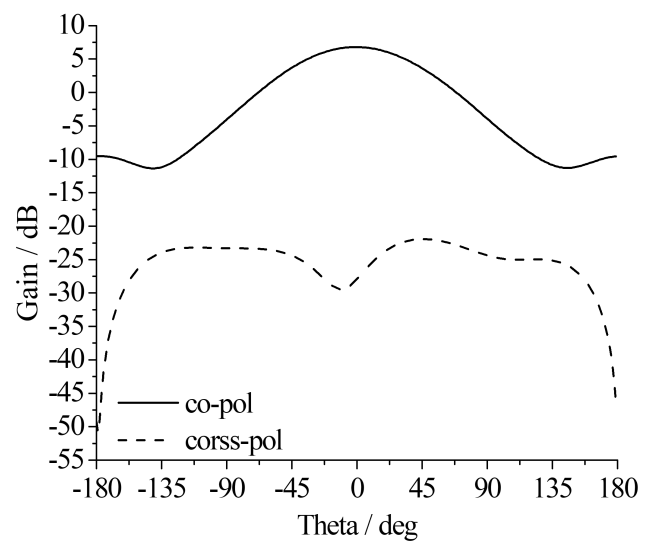

(a)

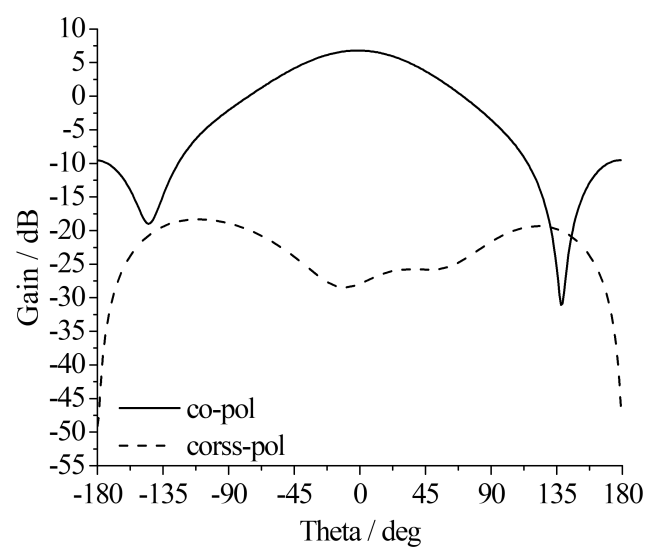

(b)

Fig. 5. The simulated radiation patterns of the designed C-shaped patch antennas with anisotropic substrate in (a) $H$-plane; and (b) $E$ plane.

the C-shaped patch antennas with anisotropic and isotropic substrates, respectively.

The radiation patterns of the C-shaped patch antennas with anisotropic and isotropic substrates are shown in Figs. 5 and 6, respectively. As shown in Fig. 5, the gain of the antenna is $6.8 \mathrm{~dB}$ and the cross-polarization is $-28 \mathrm{~dB}$. The radiation patterns in $H$-plane (yoz plane) and $E$-plane (xoz plane) are shown in Figs. 5(a) and 5(b). In Fig. 5(a), the maximum value of the cross-polarization is $-22 \mathrm{~dB}$. As shown in Fig. 5(b), the maximum value of the crosspolarization is $-18 \mathrm{~dB}$. Thus, in the whole angular span, the cross-polarization is below $-25 \mathrm{~dB}$ compared to the co-polarized gain for both $H$ - and $E$-planes. Such an omnidirectional angular span is significantly more advanced compared to the widely used DGS methods.

To demonstrate the cross-polarization suppression of the proposed method, a C-shaped patch antenna with the same dimensions is simulated and compared to the antenna with the anisotropic substrate.

The simulated radiation patterns of the C-shaped patch antenna with isotropic substrate are shown in Fig. 6. Due to 


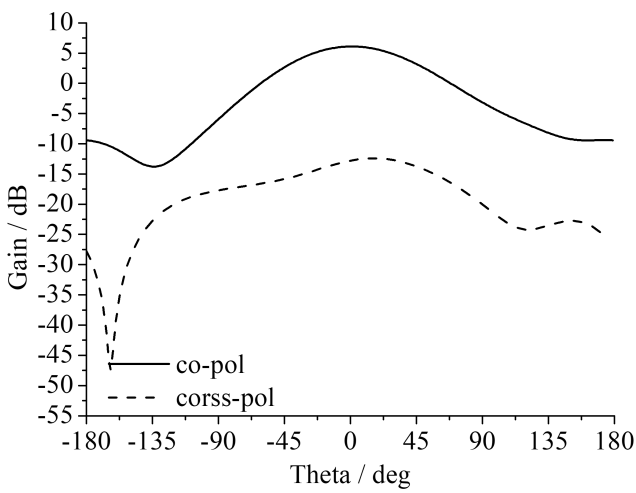

(a)

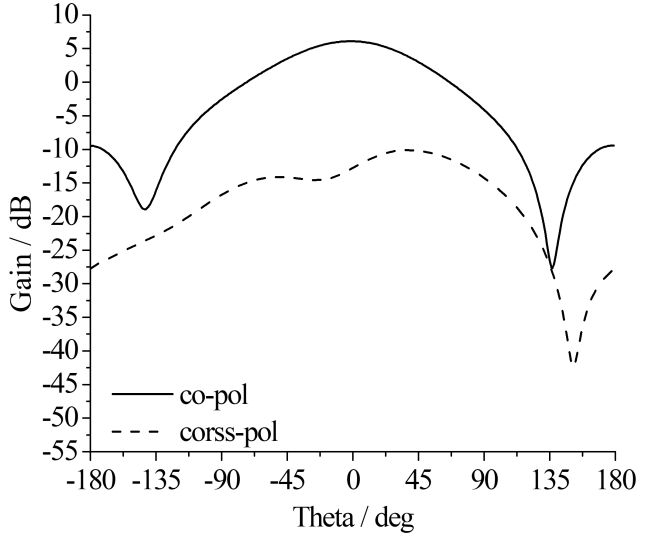

(b)

Fig. 6. The simulated radiation patterns of the designed C-shaped patch antennas with isotropic substrate in (a) $H$-plane; and (b) $E$-plane.

Table 1. Comparison of cross-polarization.

\begin{tabular}{|c|c|c|}
\hline$\theta[\mathrm{deg}]$ & $\begin{array}{c}H \text {-plane } \\
\text { isotropic/anisotropic }[\mathrm{dB}]\end{array}$ & $\begin{array}{c}E \text {-plane } \\
\text { isotropic/anisotropic }[\mathrm{dB}]\end{array}$ \\
\hline 90 & $-20 /-24.35$ & $-14.2 /-21.7$ \\
\hline 45 & $-13.7 /-22$ & $-10 /-25.8$ \\
\hline 0 & $-12.8 /-28$ & $-12.8 /-28$ \\
\hline-45 & $-15.8 /-24.4$ & $-14 /-24$ \\
\hline-90 & $-17.7 /-23.3$ & $-16.7 /-18.9$ \\
\hline
\end{tabular}

the asymmetric topology, the cross-polarization level of the $\mathrm{C}$-shaped patch antenna is greatly higher than the typical rectangular patch antenna. ${ }^{10}$

As shown in Fig. 6, the gain and cross-polarization of the C-shaped patch antenna with isotropic substrate are $6.1 \mathrm{~dB}$ and $-12.8 \mathrm{~dB}$. The maximum value of the cross-polarization component is $-12.4 \mathrm{~dB}$ and $-10 \mathrm{~dB}$ in $H$ - and $E$-planes, respectively. In Fig. 6(a), compared to the co-polarized gain, the cross-polarization is below $-18.5 \mathrm{~dB}$ in $H$-plane. The maximum value of the cross-polarization compared to the copolarized gain is $16.2 \mathrm{~dB}$ in $E$-plane, as shown in Fig. 6(b). Thus, for the antenna with anisotropic substrate, in the radiation direction, the cross component level is significantly reduced by $15.2 \mathrm{~dB}$, and the co-polarized gain is enhanced gently by $0.7 \mathrm{~dB}$.

To further discuss the data in Figs. 5 and 6, Table 1 shows a more clear comparison of the cross-polarization component of these two antennas. The cross-polarization of the $\mathrm{C}$-shaped patch antenna is suppressed in a wide angular span. At the main radiation direction $\left(\theta=0^{\circ}\right)$, the cross-polarization is suppressed greatly by $15.2 \mathrm{~dB}$. According to Table 1 , in $H$ and $E$-planes, the cross component is reduced at least by $8.3 \mathrm{~dB}$ and $10 \mathrm{~dB}$ from $45^{\circ}$ to $-45^{\circ}$, and by $4.35 \mathrm{~dB}$ and $2.2 \mathrm{~dB}$ from $90^{\circ}$ to $-90^{\circ}$, respectively.

\section{Conclusion}

In this paper, a novel method for cross-polarization suppression in a compact $\mathrm{C}$-shaped patch antenna is proposed. By applying an anisotropic substrate, additional crosspolarized field is induced and cancels the cross-polarization generated by the asymmetric patch. Thus, the cross-polarization level in the C-shaped patch antenna can be significantly reduced which makes such a compact antenna much more practical. This method has potential for cross-polarization reduction in other patch antennas with asymmetric structures.

\section{Acknowledgments}

This work was supported in part by the National Natural Science Foundation of China under Grant 61501365, 61601360, 61471292, 61331005, and the China Postdoctoral Science Foundation under Grant 2015M580849.

\section{References}

${ }^{1}$ K. F. Lee and K. M. Luk, Microstrip Patch Antennas (Imperial College Press, UK, 2010).

${ }^{2}$ D. Guha, C. Kumar and S. Pal, Improved cross-polarization characteristics of circular microstrip antenna employing arcshaped defected ground structure (DGS), IEEE Antennas Wirel. Propag. Lett. 8, 1367 (2009).

${ }^{3}$ D. Guha, M. Biswas and Y. M. M. Antar, Microstrip patch antenna with defected ground structure for cross polarization suppression, IEEE Antennas Wirel. Propag. Lett. 4, 455 (2005).

${ }^{4}$ C. Kumar and D. Guha, Asymmetric geometry of defected ground structure for rectangular microstrip: A new approach to reduce its cross-polarized fields, IEEE Trans. Antennas Propag. 64, 2503 (2016).

${ }^{5}$ W. H. Hsu and K. L. Wong, Broad-band probe-fed patch antenna with a U-shaped ground plane for cross-polarization reduction, IEEE Trans. Antennas Propag. 50, 352 (2002). 
${ }^{6}$ C. K. Ghosh, B. Mandal, B. Rana and S. K. Parui, Reduction of cross-polarization radiation of E-shaped microstrip antenna array using spiral-ring resonator, 5th Int. Conf. Computers and Devices for Communication (2012), pp. 1-4.

${ }^{7}$ H. Zhou, W. Hong, L. Tian and L. Cheng, Cross-polarization suppressed and linearly polarized patch array antenna with cavitybacked slot feed for vehicle applications, IEEE Antennas Wirel. Propag. Lett. 15, 126 (2016).

${ }^{8} \mathrm{X}$. Zhang and L. Zhu, Patch antennas with loading of a pair of shorting pins toward flexible impedance matching and low cross polarization, IEEE Trans. Antennas Propag. 64, 1226 (2016).

${ }^{9}$ G. Kossiavas, A. Papiernik, J. P. Boisset and M. Sauvan, The Cpatch — A small microstrip element, Electron. Lett. 64, 253 (1989).

${ }^{10} \mathrm{~S}$. Bhardwaj and Y. Rahmat-Samii, C-shaped, E-shaped and U-slotted patch antennas: Size, bandwidth and cross-polarization characterizations, 6th European Conf. Antennas Propag. (2012), pp. 1674-1677.
${ }^{11}$ B. Wood, J. B. Pendry and D. P. Tsai, Directed subwavelength imaging using a layered metal-dielectric system, Phys. Rev. B 64, 115116 (2006).

${ }^{12}$ J. M. Zhao, L. H. Zhang, J. S. Li, Y. J. Feng, A. Dyke, S. Hao and Y. Hao, A wide-angle multi-octave broadband waveplate based on field transformation approach, Sci. Rep. 5, 17532 (2015).

${ }^{13}$ F. Liu, Z. X. Liang and J. S. Li, Manipulating polarization and impedance signature: A reciprocal field transformation approach, Phys. Rev. Lett. 111, 033901 (2013).

${ }^{14}$ H. Y. Shi, A. X. Zhang, S. Zheng, J. X. Li and Y. S. Jiang, Dualband polarization angle independent 90 degrees polarization rotator using twisted electric-field-coupled resonators, Appl. Phys. Lett. 104, 034102 (2014).

${ }^{15}$ H. Y. Shi, J. X. Li, A. X. Zhang, J. F. Wang and Z. Xu, Broadband cross polarization converter using plasmon hybridizations in a ring/disk cavity, Opt. Express 22, 20973 (2014). 